\title{
Developmental pathways of pulmonary abnormalities in rheumatoid arthritis according to sequential HRCT findings
}

\section{Ayae Tanaka}

Dokkyo Ika Daigaku

Kazuhiro Kurasawa ( $\nabla$ kurasawa@dokkyomed.ac.jp )

Dokkyo Ika Daigaku

Sayo Soda

Dokkyo lka Daigaku

Yuta Takamura

Dokkyo Ika Daigaku

Tomoyuki Miyao

Dokkyo Ika Daigaku

Anna Hasegawa

Dokkyo Ika Daigaku

Tomoka Hiyama

Dokkyo Ika Daigaku

Ryutaro Yamazaki

Dokkyo Ika Daigaku

\section{Satoko Arai}

Dokkyo Ika Daigaku

Takayoshi Owada

Dokkyo Daigaku

Masafumi Arima

Dokkyo Ika Daigaku

Reika Maezawa

Dokkyo Ika Daigaku

\section{Research article}

Keywords: Rheumatoid arthritis, pulmonary abnormalities, developmental pathways, airway disease, interstitial lung diseases, HRCT

Posted Date: January 2nd, 2020 
DOI: https://doi.org/10.21203/rs.2.19850/v1

License: (c) (1) This work is licensed under a Creative Commons Attribution 4.0 International License. Read Full License 


\section{Abstract}

\section{Background}

To determine the patterns and development pathways of pulmonary abnormalities in patients with rheumatoid arthritis (RA).

\section{Methods}

We conducted a retrospective cohort study of consecutive RA patients who underwent high-resolution CT (HRCT) before and during biologic therapy. The presence of and change in 20 pulmonary lesions were recorded. Patterns of pre-existing and new lesions were examined by cluster analysis and tested for a correlation between pre-existing and new lesions.

\section{Results}

The subjects were 208 patients (mean age, 59.2 years; mean disease duration, 7.9 years). The mean duration between HRCT examinations was 3.3 years. Pulmonary abnormalities were found in $70 \%$ of the patients: interstitial lung disease (ILD) in $39 \%$, nodular lesions in $22 \%$, and airway disease (AD) in $55 \%$. There were commonly several different lesions in the same patient, and coexistence patterns were detected. In patients with abnormalities, AD was a shared abnormality. The incidence of pulmonary abnormalities was 10.5/100 person-years. Bronchiolitis was identified as the initial lesion. In patients with pre-existing abnormalities, a variety of new abnormalities developed in several patterns; e.g., ILD and progression of $A D$ occurred in patients with $A D$. Strong correlation was found between new ground-glass opacity and each of pre-existing $A D$ and honeycombing.

\section{Conclusions}

Pulmonary abnormalities exist and develop in patterns rather than at random. Based on the present findings, we propose the following development pathway for pulmonary abnormalities in RA: the initial lesion is bronchiolitis, which leads to the formation of complex $A D$, from which a variety of pulmonary lesions arise, particularly ILDs.

\section{Background}

Rheumatoid arthritis (RA) is a systemic autoimmune disease characterized by destructive polyarthritis. As well as joints, RA affects numerous organs, including the lungs. Pulmonary involvement contributes to the morbidity and mortality of RA patients (1-3) and is one of the major causes of death in RA (4). The presence of pulmonary abnormalities restricts the treatment of RA. Thus, the management of pulmonary involvement in RA and the treatment of RA in patients with pulmonary involvement are important.

Pulmonary involvement occurs in $60-80 \%$ of patients with RA $(5,6)$ and can affect any component of the lung. It presents as various forms of pulmonary abnormality, including interstitial lung disease (ILD), 
airway disease (AD), nodules, pleural disease, and vascular disease $(1,2)$. It is not uncommon for multiple abnormalities to be present in the same individual. To better manage these conditions, it is necessary to understand how pulmonary abnormalities develop; however, little is known regarding their developmental pathways. In the present study, we attempted to determine the developmental pathways by evaluating the co-existence patterns of pulmonary abnormalities and the relationship between pre-existing and newly emerging lesions in consecutive patients with RA.

\section{Material And Methods}

\section{Study design}

We conducted a retrospective cohort study to determine the developmental pathways in RA by evaluating the existence patterns of pulmonary abnormalities and the relationship between pre-existing and newly emerging lesions in RA patients.

\section{Patients}

The participants were 410 consecutive Japanese patients with RA who first received biologic therapy at Dokkyo Medical University Hospital between February 2004 and September 2015. All patients fulfilled the American College of Rheumatology (ACR) 1987 revised criteria for the diagnosis of RA (7) or the ACR/ The European League Against Rheumatism (EULAR) classification criteria 2010 (8) and underwent highresolution chest computed tomography (HRCT) before starting biologic therapy.

Of the 410 patients, 208 who had undergone sequential chest HRCT during biologic therapy were admitted to the study. Excluded were patients with a pulmonary infection such as bacterial pneumonia, tuberculosis, non-tuberculous mycobacterial infection and pneumocystis pneumonia or drug-induced pulmonary injury on the sequential HRCT. The data of these patients were selected for further analysis. This study was approved by the local Ethics Committee Bioethics Committee Dokkyo Medical University (\#2141).

\section{Clinical data collection}

We reviewed the subjects' medical records and collected the following data: patient characteristics, including age, sex and smoking history; respiratory symptoms and disease duration; serum levels of anticitrullinated protein antibody, rheumatoid factor (RF), and CRP; disease activity as assessed by the Disease Activity Score-28/Erythrocyte sedimentation rate (DAS28-ESR) (9); medication for RA, including glucocorticoids, conventional disease-modifying antirheumatic drugs such as MTX and biologics; and reasons for the sequential HRCT examination.

\section{Evaluation of chest HRCT images}

All subjects received HRCT of the chest before and during biologic therapy. HRCT images were evaluated independently by a rheumatologist (AT) with 10 years of experience who had also received training as a 
pulmonologist, and a pulmonologist (SS) with 11 years of experience.

The readers were blinded to all clinical information. They evaluated the existence and distribution of lung abnormalities on HRCT; abnormalities detected at the first HRCT were evaluated for any change at each subsequent scan. If the assessment differed between the two examiners, the final judgment was made by a pulmonologist (MA) with 30 years of experience, referring to reports by pulmonary radiologists.

Pulmonary abnormalities were classified into 4 categories and 20 lesion types as follows: ILD (groundglass opacity [GGO], consolidation, halo sign, reticular pattern, interlobular septal thickening, bronchovascular bundle thickening, curved opacity, linear opacity), nodular lesions (nodular pattern, small nodular pattern), AD (bronchiolitis, bronchial wall thickening, bronchiectasis, cystic bronchiectasis), and others (low attenuation area (LAA), pleural thickening, pleural calcification, pleural effusion, lymph node calcification, and atelectasis) $(10,11)$. The readers recorded the presence or absence of these 20 lesions on HRCT in each of 6 lung field regions (right/left upper, middle, and lower) and described any change in appearance of the lesions.

Change in a pulmonary lesion was defined as follows: 1) when a lesion not seen in any lung fields on the first HRCT was detected on the sequential scan, the lesion was judged as new; 2) when a lesion found on the first HRCT showed expansion within the same lung field on the sequential scan, or was newly detected in another lung field, the lesion was judged as worsened, when a lesion worsened in one lung field and was unchanged or improved in other fields, the lesion was judged as worsened.; 3) when a lesion seen on the first HRCT scan decreased in size, with no new or worsening lesions in any other lung fields, the lesion was judged as improved, when a lesion improved in one lung field and was unchanged in other fields, the lesion was judged as improved.; and 4) when a lesion seen on the HRCT scan did not change in size or distribution in all lung fields, the abnormality was judged as unchanged.

A change in category and pulmonary abnormalities was similarly defined as follows: 1 ) when a category/abnormality had a new lesion, the category/abnormality was judged as having a new lesion; 2) when a category/abnormality had a worsened lesion and no new ones, the category was judged as worsened; 3 ) when a category/ abnormality had an improved lesion and no new or worsened ones, the category was judged as improved; and 4) when a category/abnormality had no new, emerging, worsened, or improved lesions, the category/abnormality was judged as unchanged.

\section{Statistical analysis}

All analyses were performed using JMP11 software (SAS Institute Japan, Tokyo, Japan). Normally distributed continuous data were analyzed using 2-sample t-test or one-way analysis of variance and were presented as the mean \pm standard deviation (SD). Non-normally distributed continuous data were analyzed using Wilcoxon/Kruskal-Wallis test. Categorical data were analyzed using chi-square test or Fisher's exact test. For multiple comparisons, Bonferroni correction was applied. Statistical significance was defined as $p<0.05$. 
Cluster analysis was conducted according to the existence of the lesions by Ward's method. The characteristics of clusters were determined by chi-square test or Fisher's exact test followed by residual analysis (12).

To detect the correlation between pre-existing and new lesions, we conducted 4 analyses, for the reason that the numbers in the cells were small and the power to detect correlation was not strong. First, we made a checkerboard of 20 pre-existing and new lesions. Preexisting and new lesions that numbered fewer than 10 and 4, respectively, were excluded from analysis.

The following 4 analyses were conducted: 1) Fisher's exact test (presence/absence of a preexisting lesion vs. presence/absence of a new lesion); 2) determination of whether the frequency of new lesions (B) in preexisting lesions $(A)$ was beyond the $95 \%$ confidence Interval $(\mathrm{Cl})$ of the frequency of new lesions

(B) in all patients ( $B$ in $A$ vs. $B$ in total); 3 ) determination of whether the frequency of new lesions $(B)$ in patients with preexisting lesions $(A)$ and any new lesion was beyond the $95 \% \mathrm{Cl}$ of the frequency of new lesions (B) in the total patients with any new lesions ( $B$ in $A$ with new lesions vs. $B$ in total with new lesions); and 4) determination of whether the frequency of pre-existing detected lesions (A) in patients with detected new lesions (B) was beyond the $95 \% \mathrm{Cl}$ of the frequency of pre-existing detected lesions (A) in all patients with any new lesions ( $A$ in $B$ vs $A$ in total with new lesions).

\section{Results}

\section{Patient characteristics}

The subjects were 144 females (69.2\%), the age at entry was $59.25 \pm 13.16$ years, and $46.8 \%$ had a history of smoking (Table 1). The age of RA onset was $51.31 \pm 15.26$ years and disease duration was $7.94 \pm 9.31$ years. Anti-CCP antibody and RF were positive in $85.9 \%$ and $84.1 \%$ of the subjects, respectively; and there was usage of MTX and glucocorticoids in $65.3 \%$ and $75.4 \%$ of the subjects, respectively. Twenty-one patients (10.1\%) displayed respiratory symptoms (e.g., cough). The mean interval between HRCT scans obtained before and during biologic therapy was 3.26 years. The patients underwent sequential HRCT scans for the following reasons: follow-up or screening for chest abnormalities without respiratory manifestations ( $n=120 ; 58 \%)$, new/worsened symptoms $(n=46 ; 22 \%)$, new/worsened radiographic findings $(n=37 ; 18 \%)$ and abnormal laboratory findings $(n=19 ; 9 \%)$.

\section{Prevalence of pulmonary abnormalities prior to initiation of biologic therapy}

Pulmonary abnormalities were found in 146 of the 208 patients (70.2\%): ILD (38.9\%), nodular lesions (21.6\%), and AD (55.3\%) (Table 2). The most common ILD lesions were reticular pattern $(20.2 \%$ of all patients), honeycombing (6.7\%), GGO (6.3\%), and consolidation (7.7\%). Regarding nodular lesions, nodular pattern was observed in $14.4 \%$ and small nodular pattern in $12.0 \%$ of all patients. Regarding AD, bronchiolitis was observed in $40.4 \%$ and bronchiectasis in $41.3 \%$. An area of low attenuation was detected in $13.0 \%$ of patients. Commonly, several pulmonary lesions coexisted in the same individual. 
The agreement rate and the kappa statistic between the two evaluators regarding detection of lesions were 0.90 and 0.52 , respectively.

\section{Cluster analysis of the patterns of existence of pulmonary abnormalities}

Because many individuals had several pulmonary lesions, we examined the patterns of their occurrence by cluster analysis, in which clusters were determined according to the presence or absence of the 20 lesions. Six clusters were identified (Fig. 1A): cluster 1, no pulmonary abnormalities; cluster 2, bronchiolitis alone; cluster $3, A D+$ curved linear opacity; cluster $4, A D$; cluster $5, A D+$ nodular lesions; and cluster $6, A D+$ ILD (reticular pattern). Of note, AD was found in most patients with pulmonary abnormalities (clusters 2-6) suggesting that $A D$ is a shared pulmonary abnormality in patients with pulmonary lesions.

\section{Change in pulmonary abnormalities during biologic therapy}

During the observation period ( $3.3 \pm 2.6$ years), in 92 patients the pulmonary abnormalities showed change: new lesions developed in 68 , lesions improved without development of new lesions or worsening of pre-existing abnormalities in 4, and pre-existing abnormalities worsened without the development of new lesions in 20.

As shown in Table 2, the incidence of any new pulmonary lesion was $10.5 / 100$ person-years (PY). The incidence of ILD, nodular lesions, and AD was 7.0, 2.2, and 4.3/100 PY, respectively. The most common new abnormalities in ILD were GGO (3.2/100 PY) and consolidation (2.3/100 PY), and those in AD were bronchiolitis (2.3/100 PY) and bronchiectasis (2.0/100 PY).

There was much variation in the frequencies of worsening and improving lesions among the 20 lesions (Suppl. Table 1). For GGO and consolidation, some lesions worsened whereas others improved; however, some reticular pattern lesions and worsened, but few improved. Some honeycomb lesions worsened, and none improved. Lesions with small nodular pattern and AD including bronchiolitis and bronchiectasis worsened, but rarely improved.

Regarding evaluation of change in a lesion between the initial and subsequent scan, the agreement rate and the kappa statistic were 0.83 and 0.65 , respectively.

\section{Patterns of newly emerging pulmonary abnormalities}

To examine the emergence pattern of the pulmonary lesions, we conducted a cluster analysis of newly developed lesions in all 208 patients. New lesions formed clusters rather than developing at random. As shown in Fig. 1B, we identified seven clusters: cluster 1, no pulmonary abnormalities; cluster 2, nodular pattern; cluster 3, curved linear opacity; cluster 4, bronchiectasis; cluster 5, consolidation; cluster 6 , bronchiolitis; and cluster 7, GGO.

\section{Impact of preexisting pulmonary lesions on development of new abnormalities}


We examined whether the pre-existence of pulmonary abnormalities affected the development of new lesions. As shown in Fig. 2, new pulmonary abnormalities (especially ILD) frequently developed in patients with pre-existing diseases, particularly in those with nodular lesions and AD. The incidence of pulmonary lesions was 4.86/100 PY, which was lower than the incidence of pulmonary lesions $(13.5 / 100$ PY) in patients with pre-existing pulmonary abnormalities (Table 2). However, not all pulmonary lesions developed in patients with pre-existing lesions. GGO and consolidation frequently developed in patients with pre-existing disease, whereas curved linear opacity and bronchiolitis occurred in patients without pulmonary lesions (Table 2).

\section{Development of pulmonary abnormalities in patients without preexisting pulmonary abnormalities}

We attempted to determine the developmental pathways of pulmonary abnormalities in RA. First, to identify which lesions appear as initial abnormalities in RA, we examined the pulmonary lesions that developed in patients with no pre-existing abnormalities.

Of 62 patients without pulmonary abnormalities, 11 patients (17.7\%) developed a total of 23 new lesions. The most frequent lesions were bronchiolitis ( $n=6 ; 54.5 \%$ of patients with new lesions), curved linear opacity $(n=4 ; 36.4 \%)$ and bronchiectasis $(n=3 ; 36.4 \%)$ (Fig. 1C). A heat map of the lesions suggested that the initial pulmonary abnormalities were bronchiolitis with curved linear opacity/bronchiectasis (Fig. 1C). Taken together, bronchiolitis and curved linear opacity were the initial lesions.

\section{Development of pulmonary abnormalities in patients with preexisting pulmonary abnormalities}

We also examined the pulmonary lesions that developed in patients with pre-existing abnormalities. Of 146 patients with pulmonary abnormalities, 57 patients (39\%) developed a total of 92 new abnormalities. A variety of pulmonary lesions developed in these patients. A cluster analysis of new abnormalities in patients with pre-existing lesions identified five clusters: cluster 1 , no pulmonary abnormalities; cluster 2 , $A D$ with bronchiectasis/bronchial wall thickening; cluster 3 , bronchiolitis; cluster 4, consolidation; and cluster 5, GGO (Fig. 1D).

The heat map shows that clusters 2 and 3 emerged in patients with pre-existing $A D$, which suggests that $A D$ induces other $A D$ as a complex form of $A D$. Cluster 4 (consolidation) and cluster 5 (GGO) also developed in patients with pre-existing $A D$. Taken together, we consider that $A D$ induces a variety of pulmonary abnormalities, including further progression of $A D$.

\section{Correlation between preexisting and newly emerging lesions}

To examine the correlation between pre-existing and new pulmonary lesions, we performed a checkerboard analysis of pre-existing and newly emerging lesions (Suppl. Table 2). Note that many individuals had or developed several lesions.

Figure $3 \mathrm{~A}$ shows the results of the checkerboard analysis. There are several correlations between the preexisting and new lesions. There was a strong correlation of each of pre-existing honeycomb, reticular 
pattern, small nodular lesion, bronchiolitis, and bronchiectasis with new development of GGO.

The above relationships are summarized diagrammatically in Fig. 3B, which outlines the development pathways of pulmonary abnormalities in RA.

\section{Discussion}

The major findings of the present study are as follows: 1) 70\% of RA patients had pulmonary abnormalities; 2 ) at the level of the individual patient, there was coexistence of pulmonary lesions with other lesions in several patterns; 3 ) in most patients with any pulmonary lesion, AD was a shared abnormality; 4) the incidence of pulmonary abnormality was 10/100 PY; 5) the initial lesions were bronchiolitis and curved linear opacity; 6) patients with pre-existing lesions went on to develop a variety of pulmonary lesions; 7) new pulmonary lesions, especially ILD, developed frequently in patients with preexisting pulmonary abnormalities such as $\mathrm{AD}$; and 8) correlations existed between pre-existing and new lesions, and were strong between GGO and each of bronchiolitis, bronchiectasis, small nodular lesions, and honeycombing.

It is known that bronchial wall thickening is associated with bronchiolitis and that bronchiolitis, particularly follicular bronchiolitis, causes bronchial dilatation and bronchiectasis (13-15); accordingly, $A D$ lesions could cause progression to the complex form of $A D$ as each lesion in turn induces other $A D$ lesions.

In addition, interlobular septal thickening, reticular opacity, and honeycombing are radiological findings of fibrotic NSIP (16), although the latter two are also a frequent finding in usual interstitial pneumonia (UIP) (17). This suggests that these lesions form fibrotic ILD complex similar to the AD complex.

Taking the present findings together, we propose the following developmental pathways of pulmonary abnormalities in RA (Fig. 4): 1) the initial pulmonary lesions are bronchiolitis and curved linear opacity; 2) bronchiolitis progresses to complex $A D$, including bronchiectasis; 3 ) a variety of pulmonary abnormalities then arise, including AD-induced inflammatory ILDs such as GGO and consolidation, and fibrotic ILD may develop; 4) honeycombing and AD frequently lead to the development of GGO; and 5) after pulmonary lesions become established, there is development of new lesions.

This figure can also be supported by the clusters of pre-existing lesions (Fig 1A), because cluster 2 was bronchiolitis, cluster 3 was $A D$ (particularly bronchiolitis) with curved linear opacity, cluster 4 was $A D$, and clusters 5 and 6 were AD with nodules and AD with fibrotic ILD, respectively.

To the best of our knowledge, this is the first study to describe the possible sequential development pathways of pulmonary abnormalities in RA. In addition, our findings indicate the importance of $A D$ in the further development of pulmonary abnormalities in RA, both as an initial pulmonary abnormality and with regard to the variety of pulmonary lesions (particularly ILD) that arise subsequently. 
We have identified bronchiolitis and curved linear opacity as the initial lesions in RA. However, we consider that bronchiolitis was a true initial lesion and curved linear opacity was a secondary lesion because the cluster analysis revealed a cluster of bronchiolitis (cluster 2) as well as a cluster of bronchiolitis and curved linear opacity (cluster 3 ) (Fig 1A).

The present results are in agreement with those of several previous studies regarding $A D$ as initial lesions. Mori et al reported airway disease in $40 \%$ of patients with early RA (18). Demoruelle et al reported the subsequent development of RA in the $70 \%$ of their subjects who did not have arthritis but had airways disease and were positive for anti-CCP antibody (19). Similarly, a high prevalence of AD in pre- and early RA has been reported (20).

We have also shown that a variety of pulmonary lesions, particularly ILD, arose from AD. Tokuda and Takemura claimed that $A D$, particularly bronchiectasis, caused destruction of airways and dilatation of the airway tracts in RA, resulting in cystic lesions that resemble UIP. $(21,22)$. In contrast, the present findings suggested that $A D$ induced inflammatory ILD such as GGO, rather than UIP.

Although the role of $A D$ in the development of ILD remains largely unknown, various theories have been proposed. Similar to our findings, the coexistence of AD with ILD has been reported in RA $(23,24)$. In IIP, there is a type of ILD, respiratory bronchiolitis-associated ILD, which is associated with small airway inflammation (25). Moreover, recent studies found MUC5B promoter variant in patients with RA-UIP and IPF, but not in patients with RA without ILD (26). MUC5B is a mucin secreted by bronco-epithelial cells, hence a connection between AD and ILD in RA-ILD might be suggested. Furthermore, many studies have suggested the lung as a primary site where the immune response can cause RA (27). Thus, AD could induce ILD directly through the spread of inflammation at the site, and indirectly through generating the autoimmune response and systemic inflammation.

The present study showed a relationship between pre-existing honeycombing and new GGO, consistent with previous reports that RA patients frequently have UIP, which is associated with acute exacerbation of ILD and poor prognosis $(28,29)$.

The prevalence of pulmonary abnormalities, ILD, and AD in the present study was similar to that in previous studies (30). The incidence of ILD in patients without pre-existing abnormalities was 3.1/100 PY, which was high compared with previously reported cumulative incidences of $5 \%$ at 10 years (31) and $6.3 \%$ at 15 years (32). The incidence of $A D$ in patients without pulmonary abnormalities was 3.1/100 PY, which is higher than the cumulative incidence of $4 \%$ at 10 years by the study of Nannini et al (33). The difference in incidence between the present and previous reports was due to the methods employed to detect the abnormalities. In the present study, HRCT examination was conducted regardless of the patients' respiratory symptoms, whereas in previous studies ILD was diagnosed clinically.

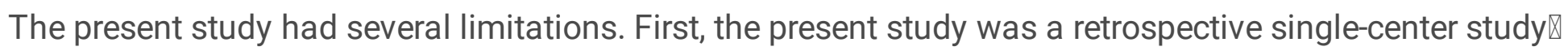
The follow-up HRCT examinations were conducted according to the decision of the physician rather than a planned schedule, and the interval between the scans varied. In addition, as $15 \%$ of patients had 
respiratory symptoms and received follow-up HRCT, there might be selection bias that caused the incidence of pulmonary abnormalities to be higher than the true incidence, although $52 \%$ of patients had no symptoms on follow-up HRCT. Second, the number of subjects was small. We did not clarify several pathways, including the development of nodular lesions. Third, our classification system of 20 lesions did not include all abnormalities because they are so numerous in RA; the glossary of the Fleischner Society describes 106 lesions (11). Fourth, because pulmonary lesions coexist with other lesions, we considered that the combination of pre-existing lesions was more important than the individual ones. However, we did not find clear relationship between pre-existing combination patterns and new lesions, which might be due to the small number of patients and methods of analysis. Lastly, the present subjects were treated with biologic therapy, which could have modified the incidence and development of new lesions.

In conclusion, we have proposed developmental pathways of pulmonary abnormalities in RA, and additionally showed the important role of $A D$ in the development of pulmonary abnormalities such as ILD. As well as helping to predict the onset of pulmonary abnormalities, which is important in the management of RA, the present study might enable development of a method for preventing the emergence and progression of pulmonary abnormalities in RA, including ILD, by addressing airway inflammation. To verify and confirm our findings, prospective multi-centered studies are required that include a large number of patients.

\section{Declarations}

\section{Availability of data and materials}

The datasets generated and/or analyzed during the current study are not publicly available for ethical and privacy reasons.

\section{Contributions}

AT and KK contributed to the study design, data analysis, data interpretation and manuscript writing. SS and SA contributed to the data analysis and data interpretation. YT, TM, AH, TH and RY contributed to the data analysis. TO and RM contributed to the study design, and data interpretation. MA contributed to the study design, data analysis, and data interpretation. All authors read and approved the final manuscript

\section{Ethics approval and consent to participate}

This study was approved by the local Ethics Committee Bioethics Committee Dokkyo Medical University (\#2141) and according to Ethical Guidelines for clinical studies 2009 by the Ministry of Education, Culture, Sports, Science and Technology and Ministry of Health, Labor and Welfare, Japan. As this was a retrospective observational cohort study, participants were not required to provide informed consent.

\section{Consent for publication}

Not applicable 


\section{Competing interests}

KK has received research support from Astellas, Asahi-Kasei, Chugai, Eisai, Mitsubishi-Tanabe, Ono and Pfizer, outside the submitted work. The other authors declare that they have no competing interests.

\section{Abbreviations}

- ACR: American College of Rheumatology

- AD: airway disease

- Cl: confidence interval

- CRP: C-reactive protein

- CT: computed tomography

- EULAR: The European League Against Rheumatism

- GGO: ground-glass opacity

- HRCT: high-resolution computed tomography

- ILD: interstitial lung disease

- LAA: low attenuation area

- MTX: methotrexate

- PY: person-years

- RA: rheumatoid arthritis

- RF: rheumatoid factor

\section{References}

1. Shaw M, Collins BF, Ho LA, Raghu G. Rheumatoid arthritis-associated lung disease. Eur Respir Rev 2015;24:1-16.

2. Yunt ZX, Solomon JJ Lung disease in rheumatoid arthritis. Rheum Dis Clin North Am 2015;41:22536.

3. Spagnolo P, Lee JS, Sverzellati N, Rossi G, Cottin V. The Lung in Rheumatoid Arthritis: Focus on Interstitial Lung Disease. Arthritis Rheumatol 2018;70:1544-1554.

4. Nakajima A, Inoue E, Tanaka E, Singh G, Sato E, Hoshi D, et al. Mortality and cause of death in Japanese patients with rheumatoid arthritis based on a large observational cohort, IORRA. Scand J Rheumatol 2010;39:360-7.

5. Cortet B, Perez T, Roux N, Flipo RM, Duquesnoy B, DelcambreB, et al. Pulmonary function tests and high resolution computed tomography of the lungs in patients with rheumatoid arthritis. Ann Rheum Dis 1997;56:596-600.

6. Turesson C, O'Fallon WM, Crowson CS, Gabriel SE, Matteson EL. Extra-articular disease manifestations in rheumatoid arthritis:incidence trends and risk factors over 46 years. Ann Rheum 
Dis 2003;62:722-7.

7. Arnett, FC, Edworthy, SM, Bloch, DA, McShane, DJ, Fries, JF, Cooper, NS, et al. The American Rheumatism Association 1987 revised criteria for the classification of rheumatoid arthritis. Arthritis Rheum 1988;31:315-24.

8. Aletaha, D, Neogi, T, Silman, AJ, Funovits, J, Felson, DT, Bingham, CO, et al. 2010 rheumatoid arthritis classification criteria: an American College of Rheumatology/ European League Against Rheumatism collaborative initiative. Arthritis Rheum 2010;62:2569-81.

9. Prevoo ML, vant Hof MA, Kuper HH, van Leeuwen MA, van de Putte LB, van Riel PL. Modified disease activity scores that include twenty-eight-joint counts. Development and validation in a prospective longitudinal study of patients with rheumatoid arthritis. Arthritis Rheum 1995;38:44-8.

10. Yamagata M, Ikeda K, Tsushima K, lesato K, Abe M, Ito T, et al. Prevalence and responsiveness to treatment of lung abnormalities on chest computed tomography in patients with microscopic polyangiitis: a multicenter, longitudinal, retrospective study of one hundred fifty consecutive hospitalbased Japanese patients. Arthritis Rheumatol 2016;68: 73-723.

11. Hansell, DM, Bankier, AA, MacMahon, H, McLoud, TC, Muller, NL, Remy, J. Fleischner Society: glossary of terms for thoracic imaging. Radiology 2008;246: 697-722.

12. Haberman SJ. The Analysis of Residuals in Cross-Classified Tables. Biometrics 1973;29:205-20.

13. Bilton D, Jones AL. Bronchiectasis: epidemiology and causes. Eur Respir Monogr 201;52: 1-10.

14. Winningham PJ, Martínez-Jiménez S, Rosado-de-Christenson ML, Betancourt SL, Restrepo CS, Eraso A. Bronchiolitis: A Practical Approach for the General Radiologist. Radiographics 2017;37:777-794.

15. Hebisawa A, Kitani M, Matsui H, Tamura A. Pathology of Airway Disease in Rheumatoid Arthritis. In: Gono T, Tokuda H, Sakai F, Takemura T. (eds) Lung Disease Associated with Rheumatoid Arthritis. Springer, Singapore 2018:33-42.

16. Johkoh T, Müller NL, Colby TV, Ichikado K, Taniguchi H, Kondoh Y, et al. Nonspecific interstitial pneumonia: correlation between thin-section CT findings and pathologic subgroups in 55 patients. Radiology 2002;225:199-204.

17. Sumikawa H, Johkoh T, Ichikado K, Taniguchi H, Kondoh Y, Fujimoto K, et al. Usual interstitial pneumonia and chronic idiopathic interstitial pneumonia: analysis of CT appearance in 92 patients. Radiology 2006;24:258-66.

18. Mori S, Cho I, Koga Y, Sugimoto M. Comparison of pulmonary abnormalities on high-resolution computed tomography in patients with early versus longstanding rheumatoid arthritis. J Rheumatol 2008;35:1513-21.

19. Demoruelle MK, Weisman MH, Simonian PL, Lynch DA, Sachs PB, Pedraza IF, et al. Brief report: airways abnormalities and rheumatoid arthritis-related autoantibodies in subjects without arthritis: early injury or initiating site of autoimmunity? Arthritis Rheum 2012;64:1756-61.

20. Reynisdottir G, Karimi R, Joshua V, Olsen H, Hensvold AH, Harju A, et al. Structural changes and antibody enrichment in the lungs are early features of anti-citrullinated protein antibody-positive rheumatoid arthritis. Arthritis Rheumatol 2014;66:31-9. 
21. Tokuda H. Comprehensive Understanding of Airway Disease in Rheumatoid Arthritis. In: Gono T, Tokuda H, Sakai F, Takemura T. (eds) Lung Disease Associated with Rheumatoid Arthritis. Springer, Singapore 2018:11-32.

22. Takemura T. Pathology of Interstitial Lung Disease in Patients with Rheumatoid Arthritis. In: Gono T, Tokuda H, Sakai F, Takemura T. (eds) Lung Disease Associated with Rheumatoid Arthritis. Springer, Singapore 2018:91-116.

23. Cortet B1, Perez T, Roux N, Flipo RM, Duquesnoy B, Delcambre B, et al. Pulmonary function tests and high resolution computed tomography of the lungs in patients with rheumatoid arthritis. Ann Rheum Dis 1997;56:596-600.

24. Wilsher M1, Voight L, Milne D, Teh M, Good N, Kolbe J, et al. Prevalence of airway and parenchymal abnormalities in newly diagnosed rheumatoid arthritis. Respir Med 2012;106:1441-6.

25. Vassallo R, Ryu JH. Tobacco smoke-related diffuse lung diseases. Semin Respir Crit Care Med 2008;29:643-650.

26. Juge PA, Lee JS, Ebstein E, Furukawa H, Dobrinskikh E, Gazal S, et al. MUC5B Promoter Variant and Rheumatoid Arthritis with Interstitial Lung Disease. N Engl J Med 2018;379:2209-2219.

27. Holers VM, Demoruelle MK, Kuhn KA, Buckner JH, Robinson WH, Okamoto Y, et al. Rheumatoid arthritis and the mucosal origins hypothesis: protection turns to destruction. Nat Rev Rheumatol 2018;14:542-557.

28. Kim EJ, Elicker BM, Maldonado F, Webb WR, Ryu JH, Van Uden JH, et al. Usual interstitial pneumonia in rheumatoid arthritis-associated interstitial lung disease. Eur Respir J 2010;35:1322-8.

29. Hozumi H, Nakamura Y, Johkoh T, Sumikawa H, Colby TV, Kono M, et al. Acute exacerbation in rheumatoid arthritis-associated interstitial lung disease: a retrospective case control study. BMJ Open 2013;13;3:e003132.

30. Demoruelle MK, Olson AL, Solomon JJ. The Epidemiology of Rheumatoid Arthritis-Associated Lung Disease. In: Fischer A, Lee SJ. (eds) Lung Disease in Rheumatoid Arthritis. Humana Press, Cham, Switzerland. 2018:45-58.

31. Myasoedova E, Crowson CS, Turesson C, Gabriel SE, Matteson EL. Incidence of extraarticular rheumatoid arthritis in Olmsted County, Minnesota, in 1995-2007 versus 1985-1994: a populationbased study. J Rheumatol 2011;38:983-9.

32. Koduri G, Norton S, Young A, Cox N, Davies P, Devlin J, et al. Interstitial lung disease has a poor prognosis in rheumatoid arthritis: results from an inception cohort. Rheumatology (Oxford) 2010;49:1483-9.

33. Nannini C, Medina-Velasquez YF, Achenbach SJ, Crowson CS, Ryu JH, Vassallo R, et al. Incidence and mortality of obstructive lung disease in rheumatoid arthritis: a population-based study. Arthritis Care Res (Hoboken) 2013;65:1243-50.

\section{Tables}


Due to technical limitations, the tables have been placed in the Supplementary Files section.

\section{Note}

Figure 3B was not included in this submission.

\section{Figures}

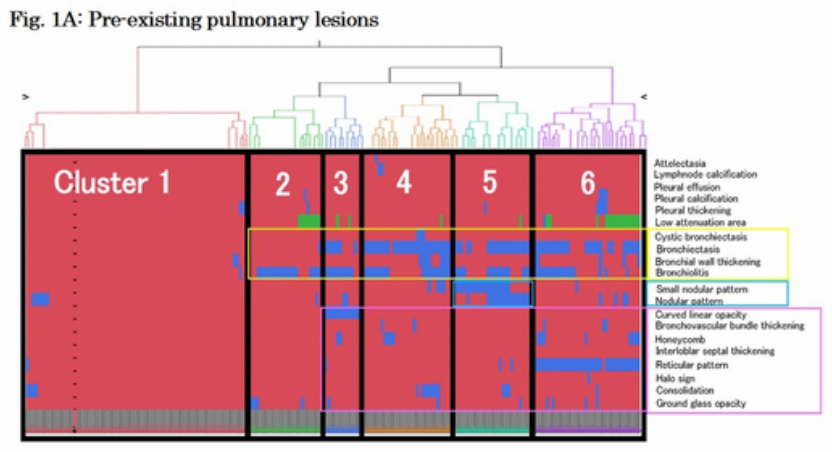

Fig. 1B: Newly emerging pulmonary lesions

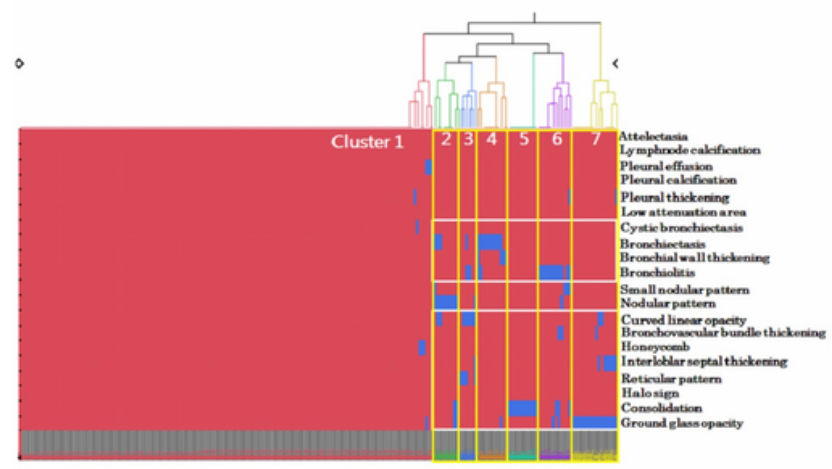

Fig. 1C; New lesions in patients without pre-existing pulmonary lesions

Fig. 1D; New lesions in patients with pre-existing pulmonary lesions
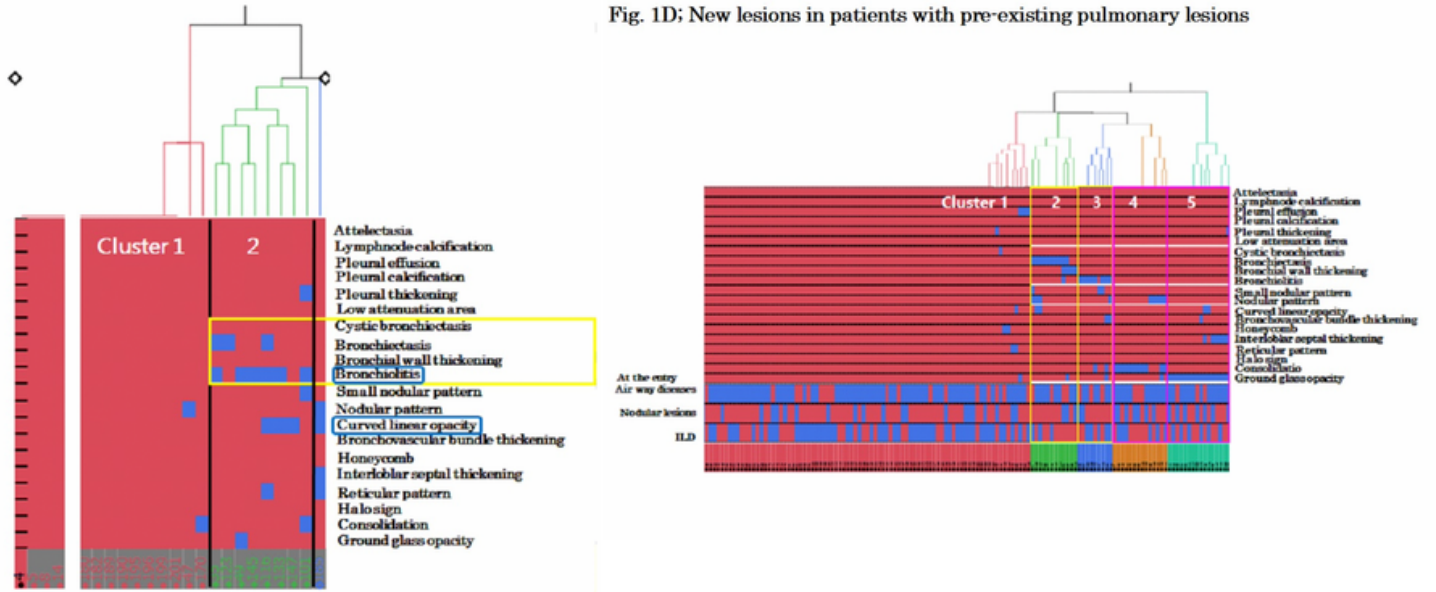

\section{Figure 1}

Patterns of pre-existing and new pulmonary abnormalities Clusters determined according to the presence or absence of the 20 lesions prior to initiation of biologic therapy (A); at sequential HDCT examination during biologic therapy (B); at sequential HDCT examination in patients without pre-existing pulmonary abnormalities (C); and at sequential HDCT examination in patients with pre-existing pulmonary abnormalities (D). Blue cells, presence; red cells, absence. 
Fig. 2.

Newly emerging abnormalities
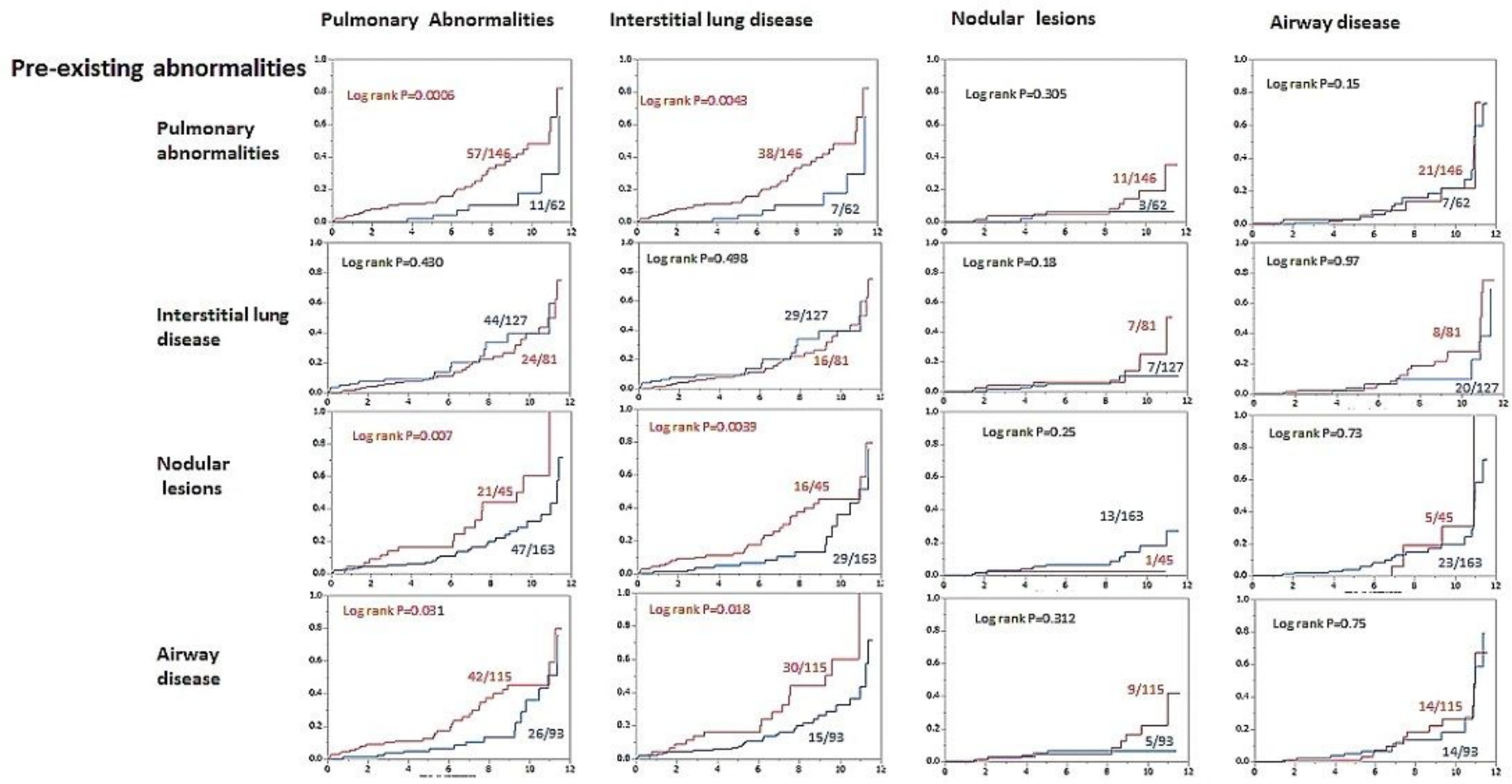

\section{Figure 2}

Cumulative incidence rates of new pulmonary abnormalities in patients with pre-existing abnormalities Red line, pre-existing abnormality (+); blue line, no pre-existing abnormality (-). ILD, interstitial lung disease; $A D$, airway disease. 
Fig. $3 \mathrm{~A}$

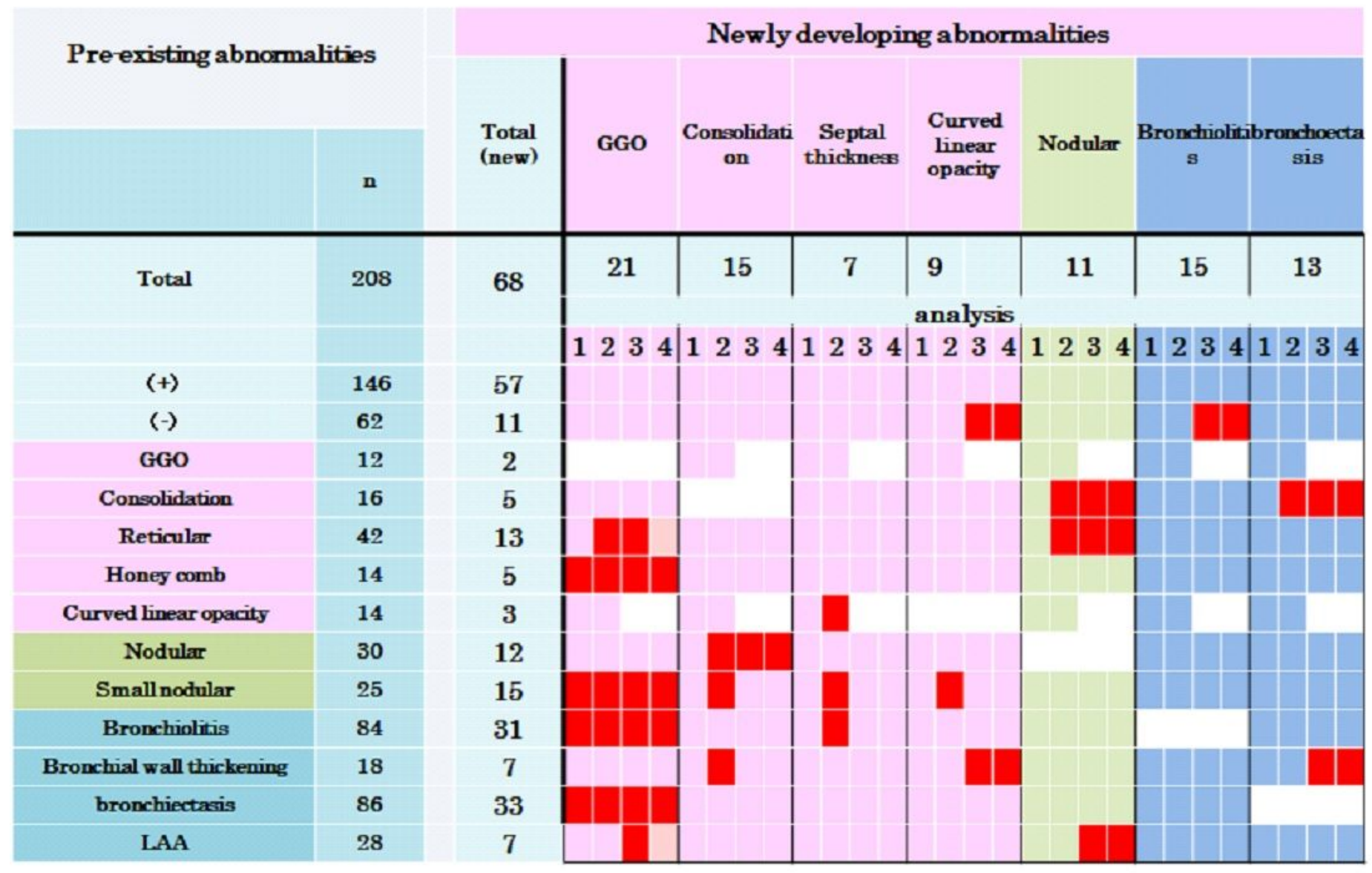

Figure 3

Correlation between pre-existing and new pulmonary abnormalities A: Summary of checkerboard analysis. Cells were included in the analysis when the number of total patients with preexisting abnormalities $>10$, and the number of total patients with new abnormalities $>4$. White cells (insufficient number of patients) were not included in the analysis. Four analyses were conducted, as described in the Material and Methods. Cells colored red indicate a positive correlation between pre-existing and new abnormalities, for each of the four analyses. B: Relation of pre-existing lesions to new abnormalities according to the results of checkerboard analysis. The red, black, blue, and brown lines indicate relations with $4,3,2$, and 1 positive results, respectively, in the four analyses. 
Fig.4

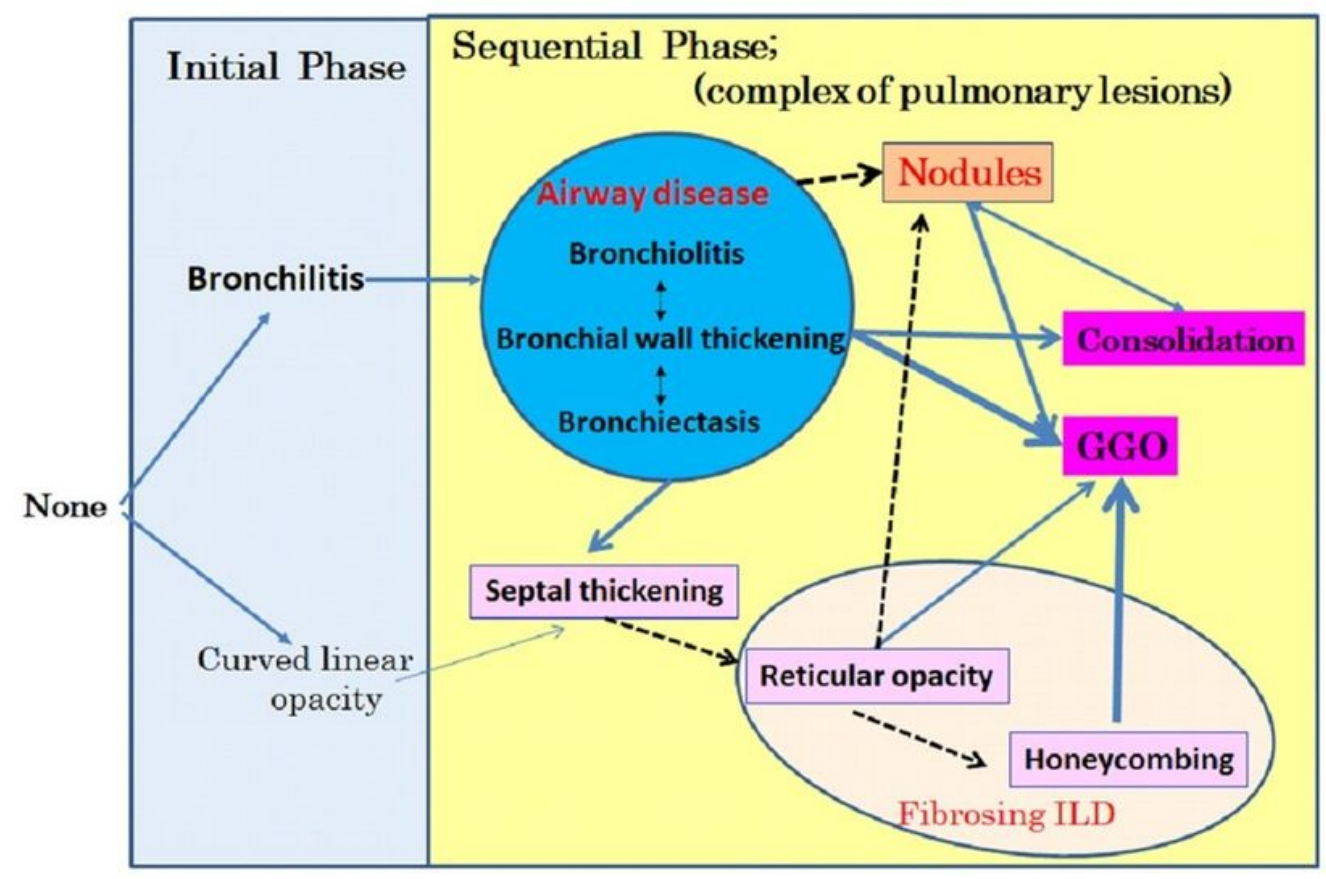

\section{Figure 4}

Proposed developmental pathways of pulmonary abnormalities in rheumatoid arthritis

\section{Supplementary Files}

This is a list of supplementary files associated with this preprint. Click to download.

- Stable1TanakaART20191225.docx

- STable2TanakaART20191225.docx 